\title{
Scattering analysis of two-dimensional electrons in AIGaN/GaN with bulk related parameters extracted by simple parallel conduction extraction method
}

S. B. Lisesivdin, A. Yildiz, N. Balkan, M. Kasap, S. Ozcelik, and E. Ozbay

Citation: Journal of Applied Physics 108, 013712 (2010);

View online: https://doi.org/10.1063/1.3456008

View Table of Contents: http://aip.scitation.org/toc/jap/108/1

Published by the American Institute of Physics

\section{Articles you may be interested in}

Two-dimensional electron gases induced by spontaneous and piezoelectric polarization charges in N- and Gaface $\mathrm{AIGaN} / \mathrm{GaN}$ heterostructures Journal of Applied Physics 85, 3222 (1999); 10.1063/1.369664

Two dimensional electron gases induced by spontaneous and piezoelectric polarization in undoped and doped AlGaN/GaN heterostructures

Journal of Applied Physics 87, 334 (1999); 10.1063/1.371866

Electron mobility, Hall scattering factor, and sheet conductivity in AlGaN/AIN/GaN heterostructures Journal of Applied Physics 110, 113713 (2011); 10.1063/1.3665124

Polarization-induced charge and electron mobility in AIGaN/GaN heterostructures grown by plasma-assisted molecular-beam epitaxy

Journal of Applied Physics 86, 4520 (1999); 10.1063/1.371396

Polarization effects, surface states, and the source of electrons in AIGaN/GaN heterostructure field effect transistors

Applied Physics Letters 77, 250 (2000); 10.1063/1.126940

Charge control and mobility studies for an $\mathrm{AlGaN} / \mathrm{GaN}$ high electron mobility transistor Journal of Applied Physics 85, 587 (1998); 10.1063/1.369493

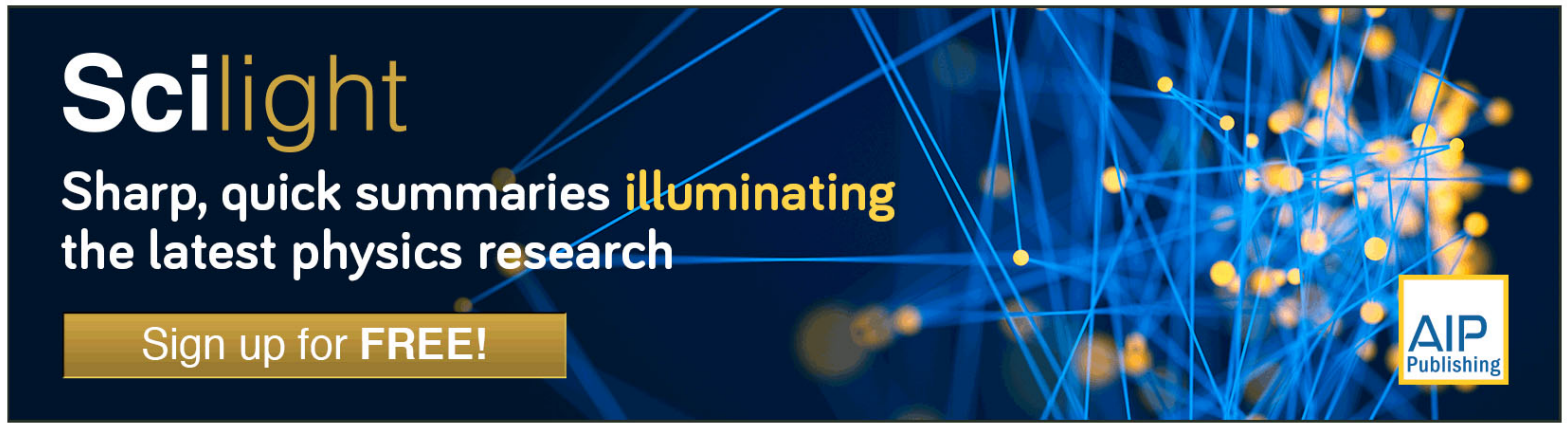




\title{
Scattering analysis of two-dimensional electrons in AIGaN/GaN with bulk related parameters extracted by simple parallel conduction extraction method
}

\author{
S. B. Lisesivdin, ${ }^{1,2}$ A. Yildiz, ${ }^{3,4}$ N. Balkan, ${ }^{5}$ M. Kasap, ${ }^{2}$ S. Ozcelik, ${ }^{2}$ and E. Ozbay ${ }^{1,6}$ \\ ${ }^{1}$ Nanotechnology Research Center, Bilkent University, Bilkent, 06800 Ankara, Turkey \\ ${ }^{2}$ Department of Physics, Faculty of Science and Arts, Gazi University, Teknikokullar, 06500 Ankara, Turkey \\ ${ }^{3}$ Department of Physics, Faculty of Science and Arts, Ahi Evran University, Aşılkpaşa Kampüsü, \\ 40040 Kirsehir, Turkey \\ ${ }^{4}$ Department of Engineering Physics, Faculty of Engineering, Ankara University, Besevler, 06100 Ankara, \\ Turkey \\ ${ }^{5}$ School of Computer Science and Electronic Engineering, University of Essex, CO4 3SQ Colchester, United \\ Kingdom \\ ${ }^{6}$ Department of Physics, Bilkent University, Bilkent, 06800 Ankara, Turkey and Department of Electrical \\ and Electronics Engineering, Bilkent University, Bilkent, 06800 Ankara, Turkey
}

(Received 23 November 2009; accepted 21 May 2010; published online 15 July 2010)

We carried out the temperature $(22-350 \mathrm{~K})$ and magnetic field $(0.05$ and $1.4 \mathrm{~T})$ dependent Hall mobility and carrier density measurements on $\mathrm{Al}_{0.22} \mathrm{Ga}_{0.78} \mathrm{~N} / \mathrm{GaN}$ heterostructures with AlN interlayer grown by metal-organic chemical-vapor deposition. Hall data is analyzed with a simple parallel conduction extraction method and temperature dependent mobility and carrier densities of the bulk and two-dimensional (2D) electrons are extracted successfully. The results for the bulk carriers are discussed using a theoretical model that includes the most important scattering mechanisms that contribute to the mobility. In order to investigate the mobility of two-dimensional electron gas, we used a theoretical model that takes into account the polar optical phonon scattering, acoustic phonon scattering, background impurity scattering, and interface roughness scattering in 2D. In these calculations, the values are used for the deformation potential and ionized impurity density values were obtained from the bulk scattering analysis. Therefore, the number of fitting parameters was reduced from four to two. () 2010 American Institute of Physics. [doi:10.1063/1.3456008]

\section{INTRODUCTION}

The GaN-based high electron mobility transistors (HEMTs) have generated a considerable amount of research activity over the past decade because of their superior electronic properties in advanced high power and high temperature applications. ${ }^{1,2}$ Even in undoped samples of $\mathrm{Al}_{\mathrm{x}} \mathrm{Ga}_{1-\mathrm{x}} \mathrm{N} / \mathrm{GaN}$ based heterostructures, it is common to observe the formation of a two-dimensional electron gas (2DEG) at the interface with high sheet carrier density values. ${ }^{1,2}$ In addition to this two-dimensional (2D) conduction, impurity and/or dislocation related bulk conduction takes place in the remaining bulk $\mathrm{GaN}$ and $\mathrm{Al}_{\mathrm{x}} \mathrm{Ga}_{1-\mathrm{x}} \mathrm{N}$ layers contributing to the overall electronic conduction in these heterostructures. ${ }^{3}$ Therefore, the measured conduction is a sum of the 2D and bulk contributions. In order to understand the properties of the individual conduction channels a detailed investigation of parallel electron transport properties needs to be carried out.

The mobility and carrier density of the 2DEG and the bulk carriers in these heterostructures are the key parameters related to the device performance. There are number of papers which reported the scattering analyses of 2DEG transport properties in $\mathrm{AlGaN} / \mathrm{GaN}$ and $\mathrm{AlInN} / \mathrm{GaN}$ systems. ${ }^{4-8}$ However, these studies are based on the analysis of pure 2D or a combination of $2 \mathrm{D}$ and three-dimensional (3D) scattering processes. In our previous studies, we pointed out the inaccuracy of such analysis and reported a plausible scattering analysis for the 2DEG extracted using the quantitative mobility spectrum analysis (QMSA) from the magnetic field dependent Hall data. ${ }^{9,10}$ QMSA is a state-of-art technique to extract information about multiple conductivity channels in a material without a limit on the carrier type and number of channels. ${ }^{11,12}$ The technique allows the successful extraction of high-mobility channels like 2DEG. However, lowmobility channels cannot be extracted successfully with this method due to magnetic field dependent quenching limit $\left(\mu_{\min } B_{\max } \approx 1\right)$. Here, $\mu_{\min }$ is the minimum mobility of a carrier which can be extracted at a magnetic field $B_{\max }$.

To extract the contributions of bulk and 2DEG carriers, we developed a simple method which is called the simple parallel conduction extraction method (SPCEM). ${ }^{13}$ SPCEM can be used for the extraction of temperature dependent mobilities and carrier densities of a 2DEG carrier and a bulk carrier in HEMTs and modulation-doped field-effect transistor (MODFETs) with the use of some assumptions.

In this work, we investigated the temperature (22-350 $\mathrm{K})$ and magnetic field $(0.05$ and $1.4 \mathrm{~T})$ dependent Hall results of $\mathrm{Al}_{0.22} \mathrm{Ga}_{0.78} \mathrm{~N} / \mathrm{GaN}$-based heterostructures with an AlN interlayer and AlN buffer layer using the SPCEM. The scattering analysis of bulk transport gave the values for the ionized impurity density and deformation potential which are then used in scattering analysis of the 2DEG. Therefore, the scat- 
tering analysis of the 2DEG carrier is performed with only two adjustable fitting parameters which are normally four or five.

\section{EXPERIMENTAL TECHNIQUES}

Four identical samples from two wafers were prepared in this work. These samples were grown on identical c-face (0001) sapphire $\left(\mathrm{Al}_{2} \mathrm{O}_{3}\right)$ substrates in a low-pressure metalorganic chemical-vapor deposition (MOCVD) reactor. Before the growth, the sapphire substrates were cleaned in $\mathrm{H}_{2}$ ambient at $1100{ }^{\circ} \mathrm{C}$, and then an AlN nucleation layers were grown at $840{ }^{\circ} \mathrm{C}$. After the deposition of the AlN nucleation layer, the wafers were heated to a high temperature for annealing. Then, a $600 \mathrm{~nm}$ AlN buffer layers were deposited on the annealed nucleation layers at $1032{ }^{\circ} \mathrm{C}$. After the deposition of buffer layers, approximately $1.9 \mu \mathrm{m}$ high growthrated GaN layers were grown. Finally, $1.5 \mathrm{~nm}$ AlN interlayers, $27 \mathrm{~nm} \mathrm{Al}{ }_{0.22} \mathrm{Ga}_{0.78} \mathrm{~N}$ barrier layers and $3 \mathrm{~nm}$ GaN cap layers were grown in order. All layers are nominally undoped. Al mole fractions of the barrier layers were determined by high-resolution x-ray diffraction (XRD) measurements.

By the means of Hall measurements with van der Pauw geometry, square shaped $\left(5 \times 5 \mathrm{~mm}^{2}\right)$ samples were prepared; temperature and magnetic field dependent (at 0.05 an $1.4 \mathrm{~T}$ ) mobilities and sheet carrier densities were measured. Ohmic contacts were formed using four evaporated Ti/Al/ $\mathrm{Ni} / \mathrm{Au}$ contacts at the corners together with indium soldering to external wires. The Ohmic behavior was confirmed by the current voltage characteristics at low temperatures. The Hall measurements were taken in a temperature range of 28-350 $\mathrm{K}$ using a Lakeshore Hall effect measurement system. At temperature steps, the Hall coefficient (with maximum 5\% error in the studied range) and resistivity (with maximum $0.2 \%$ error in the studied range) were measured for both current directions, magnetic field polarizations, and all possible contact configurations. XRD and Hall measurement results of the samples represented high similarities. Therefore, results of the one of the samples are shown as example in this study. The low $(0.05 \mathrm{~T})$ and high $(1.4 \mathrm{~T})$ magnetic field dependent data has been used in SPCEM to calculate 2DEG and bulk contributions in the investigated samples.

\section{THEORY}

\section{A. SPCEM}

The analysis of Hall data for the parallel conduction problem have been discussed in many papers with using the methods like: two-carrier model, ${ }^{14}$ multicarrier fitting procedure (MCF), ${ }^{15}$ mobility-spectrum analysis (MSA), ${ }^{16} \mathrm{MCF}$ and MSA hybrid, ${ }^{17}$ and the QMSA. ${ }^{11,12}$ All these methods have advantages and disadvantages over each other depending on the individual problem that they address. To extract the contributions of bulk and 2DEG carriers in a HEMT or MODFET structure, the SPCEM expected to be more successful than the other comparable techniques due to its less magnetic field dependency and being specially derived for these types of samples. ${ }^{13}$ In the application of SPCEM analysis, some underlying assumptions are made.
(1) There are two main contributions to conductivity in a HEMT structure: 2DEG and bulk carriers.

(2) At the low temperatures, bulk carriers are assumed to be frozen. Therefore, the measured Hall carrier density at the lowest temperature is the 2DEG carrier density that remains constant in the whole range of measurement temperatures.

(3) Because the 2DEG carrier density is temperature independent; ${ }^{18}$ the change in temperature dependent measured carrier density is caused by the thermal activation of bulk carriers only.

(4) Densities of bulk carriers and the 2DEG are roughly in the same order.

With the investigation of magnetic field dependent conductivity tensors and their derivatives following equations were found for the mobilities of 2DEG carrier (named as carrier 1) and bulk carrier (named as carrier 2):

$$
\begin{aligned}
& \mu_{1} \cong \mu_{H}^{\mathrm{Lo}} \sqrt{\frac{n_{H}^{\mathrm{Lo}}}{n_{1}^{\mathrm{Lo}}}}, \\
& \mu_{2} \cong \mu_{H}^{\mathrm{Hi}} \frac{n_{H}^{\mathrm{Hi}}-n_{1}^{\mathrm{Hi}}}{n_{H}^{\mathrm{Hi}}}=\mu_{H}^{\mathrm{Hi}} \frac{n_{2}^{\mathrm{Hi}}}{n_{H}^{\mathrm{Hi}}} .
\end{aligned}
$$

Here, $\mu_{H}^{\mathrm{Lo}}, n_{H}^{\mathrm{Lo}}, \mu_{H}^{\mathrm{Hi}}, n_{H}^{\mathrm{Hi}}$ are Hall mobilities and Hall carrier densities at low magnetic fields and at high magnetic fields, respectively. For the calculation of temperature independent 2DEG carrier density, $n_{1}^{\mathrm{Lo}}=n_{H}^{\mathrm{Lo}}$ and $n_{1}^{\mathrm{Hi}}=n_{H}^{\mathrm{Hi}}$ are used at the lowest temperature available. For the bulk carrier density contribution, $n_{2}^{\mathrm{Lo}}=n_{H}^{\mathrm{Lo}}-n_{1}^{\mathrm{Lo}}$ and $n_{2}^{\mathrm{Hi}}=n_{H}^{\mathrm{Hi}}-n_{1}^{\mathrm{Hi}}$ are used.

\section{B. Scattering mechanisms}

Scattering mechanisms of bulk and 2D carriers in III-V heterojunctions are well described in Refs. 5, 19, and 20. The analytical expressions of the bulk and 2D scattering mechanisms used in our calculations are summarized below. The final mobility value of the bulk and 2D carriers have been obtained with implementing Matthiessen's rule

$$
\frac{1}{\mu}=\sum_{i} \frac{1}{\mu_{i}},
$$

for each carrier where $\mu_{i}$ is the contribution due to the $i^{\text {th }}$ scattering mechanism characterized by a scattering time equal to $\tau_{i}$, i.e., $\mu_{i}=e \tau_{i} / m^{*}$. Here, $e$ is the charge of the electron, and $m^{*}$ is the effective mass. The material parameters used in scattering analyses are listed in Table I.

\section{Scattering mechanisms used for bulk carriers}

In our calculations, three main scattering mechanisms due to phonons and impurities are taken into account.

a. Ionized impurity scattering. Ionized impurity scattering occurs at specific scattering centers. Ionized impurity scattering is an elastic process and so it is an important source of momentum relaxation. With the Brooks-Herring model, the mobility limited by ionized impurity scattering mechanism is given as ${ }^{21}$ 
TABLE I. Material constants of GaN used in scattering calculations (Refs. 19 and 20).

\begin{tabular}{lc}
\hline \hline High frequency dielectric constant & $\varepsilon_{\infty}=5.35$ \\
Static dielectric constant & $\varepsilon_{s}=8.9$ \\
Electron effective mass & $m^{*}=0.22 \mathrm{~m}_{0}$ \\
LO-phonon energy & $\hbar \omega=0.092 \mathrm{eV}$ \\
LA-phonon velocity & $u_{l}=6.56 \times 10^{3} \mathrm{~m} \mathrm{~s}^{-1}$ \\
Density of crystal & $\rho=6.15 \times 10^{3} \mathrm{~kg} \mathrm{~m}^{-3}$ \\
Electron wave vector & $k=7.3 \times 10^{8} \mathrm{~m}^{-1}$ \\
The electromechanical coupling coefficient & $K^{2}=0.039$ \\
LA elastic constant & $c_{\mathrm{LA}}=2.650 \times 10^{11} \mathrm{~N} \mathrm{~m}^{-2}$ \\
TA elastic constant & $c_{\mathrm{TA}}=0.442 \times 10^{11} \mathrm{~N} \mathrm{~m}^{-2}$ \\
\hline \hline
\end{tabular}

$$
\mu_{I I}=\sqrt{\frac{128\left(k_{B} T\right)^{3}}{m^{*} \pi^{3}}} \frac{\left(4 \pi \varepsilon_{S}\right)^{2}}{Z^{2} e^{3} N_{\mathrm{IMP}}\left[\ln \left[1+\beta^{2}\right]-\frac{\beta^{2}}{1+\beta^{2}}\right]} .
$$

Here, $Z$ which is taken as unity in our calculations is the charge number of each ionized atom, $\varepsilon_{s}$ is the static dielectric constant, $T$ is the lattice temperature, $N_{\text {IMP }}$ is the density of ionized impurities in the crystal, and

$$
\beta=\frac{2 m^{*}}{\hbar} \lambda_{D} \sqrt{\frac{2}{m^{*}} 3 k_{B} T} .
$$

Here, $\lambda_{D}$ is the three-dimensional Debye screening length which is given as ${ }^{20}$

$$
\lambda_{D}=\sqrt{\frac{k_{B} T \varepsilon_{s}}{e^{2} n}} .
$$

Here, $n$ is the bulk carrier density at the temperature $T$.

b. Polar optical phonon scattering. Because of $\mathrm{GaN}$ is highly polar material, polar interactions dominate the scattering processes at high temperatures. Therefore, inclusion of optical phonon scattering in our calculations has a great importance. For the longitudinal polar optical phonon (LOphonon) scattering, we use a simple mobility expression with a momentum relaxation time term in the form

$$
\mu_{\mathrm{PO}(\mathrm{BULK})}=\frac{e \tau_{m}}{m^{*}} e^{\hbar \omega_{\mathrm{PO}} / k T},
$$

where, $\tau_{m}$ is the momentum relaxation time and $\hbar \omega_{\mathrm{PO}}$ is the polar optical phonon energy. ${ }^{22}$

c. Acoustic phonon scattering. For the acoustic phonon scattering, we consider a scattering of electrons by bulk acoustic phonons via both the deformation potential and piezoelectric fields. The deformation potential scattering limited mobility is given by ${ }^{5}$

$$
\begin{aligned}
\mu_{\mathrm{DP}(\mathrm{BULK})}= & \frac{\pi \hbar^{3} c_{\mathrm{LA}} e}{E_{D}^{2} k_{B} T m^{* 2} k}\left[1-\frac{q_{s 3 \mathrm{D}}^{2}}{k^{2}}+\frac{q_{s 3 \mathrm{D}}^{4}}{8 k^{4}}(3 \ln [1\right. \\
& \left.\left.\left.+\left(\frac{2 k}{q_{s 3 \mathrm{D}}}\right)^{2}\right]-\frac{1}{1+\left(q_{s 3 \mathrm{D}} / 2 k\right)^{2}}\right)\right]^{-1},
\end{aligned}
$$

where $E_{D}$ is the deformation potential constant, $k$ is the electron wave vector, and $q_{s 3 \mathrm{D}}$ is the reciprocal screening length in $3 \mathrm{D}$,

$$
q_{s 3 \mathrm{D}}^{2}=-\frac{e^{2}}{\varepsilon_{s}} \int \frac{d f(E)}{d E} N(E) d(E) .
$$

Here, $N(E)$ is the density of states function and $f(E)$ is the Fermi-Dirac function. The acoustic phonon scattering via piezoelectric effect is related to the dimensionless electromechanical coupling coefficient $K$, and the limiting mobility for piezoelectric scattering is given by ${ }^{5}$

$$
\begin{aligned}
\mu_{\mathrm{PE}(\mathrm{BULK})}= & \frac{2 \pi \varepsilon_{s} \hbar^{3} k}{K^{2} e k_{B} T m^{* 2}}\left[1-\frac{q_{s 3 \mathrm{D}}^{2}}{k^{2}}+\frac{q_{s 3 \mathrm{D}}^{4}}{8 k^{4}}(3 \ln [1\right. \\
& \left.\left.\left.+\left(\frac{2 k}{q_{s 3 \mathrm{D}}}\right)^{2}\right]-\frac{1}{1+\left(q_{s 3 \mathrm{D}} / 2 k\right)^{2}}\right)\right]^{-1} .
\end{aligned}
$$
$\mathrm{as}^{23}$

Here, the electromechanical coupling coefficient is given

$$
K^{2}=\frac{\varepsilon_{\mathrm{LA}}^{2}}{\varepsilon_{s} c_{\mathrm{LA}}}-\frac{\varepsilon_{\mathrm{TA}}^{2}}{\varepsilon_{s} c_{\mathrm{TA}}},
$$

where, $\varepsilon_{\mathrm{LA}}, \varepsilon_{\mathrm{TA}}, c_{\mathrm{LA}}, c_{\mathrm{TA}}$ are the effective piezoelectric constants and the averaged elastic constants related to longitudinal and transverse acoustic phonons, respectively. The total acoustic phonon limited mobility $\left(\mu_{\mathrm{AC}(\mathrm{BULK})}\right)$ can be calculated using Eqs. (8) and (10).

$$
\frac{1}{\mu_{\mathrm{AC}(\mathrm{BULK})}}=\frac{1}{\mu_{\mathrm{DP}(\mathrm{BULK})}}+\frac{1}{\mu_{\mathrm{PE}(\mathrm{BULK})}} .
$$

\section{Scattering mechanisms used for 2DEG}

In our investigations, polar optical phonon, acoustical phonon, background impurity, and interface roughness scattering are taken into account. Other two possible 2D scattering mechanisms, i.e., alloy and charged dislocation scattering mechanisms are neglected. The investigated samples have AlN interlayers, which are used to reduce the alloy disorder scattering by minimizing the wave function penetration from the $2 \mathrm{DEG}$ channel into the AlGaN layer. ${ }^{24}$ Therefore, alloy disorder scattering ${ }^{25}$ is neglected. Furthermore, the net carrier densities of the investigated samples are very high as $\sim 5 \times 10^{19} \mathrm{~cm}^{-3}$. The transport is dominated by ionized impurity scattering because of high carrier densities. ${ }^{26}$ Here, the scatterings due to charged dislocations are also neglected.

a. Polar optical phonon scattering. The expression of the mobility limited by LO-phonon scattering for $2 \mathrm{D}$ conduction is given by Ridley as ${ }^{27}$

$$
\mu_{\mathrm{PO}}=\frac{4 \pi \varepsilon_{0} \varepsilon_{p} \hbar^{2}}{e \omega_{\mathrm{PO}} m^{* 2} Z_{0}}\left[e^{\hbar \omega_{\mathrm{PO}} / k_{B} T}-1\right],
$$

where

$$
\frac{1}{\varepsilon_{p}}=\frac{1}{\varepsilon_{\infty}}-\frac{1}{\varepsilon_{s}} .
$$

Here, $Z_{0}$ is the width of the well where the $2 \mathrm{DEG}$ is populated and $\varepsilon_{\infty}$ is the high frequency dielectric constant.

$b$. Acoustic phonon scattering. At intermediate temperatures, the contribution of low energy acoustic phonons to the overall scattering increases and becomes comparable to longitudinal optical phonon scattering. In this work, we use 
elastic acoustic phonon scattering model where we consider both deformation potential and piezoelectric scattering as we do in the bulk case. The limited mobility expression of deformation potential is given as ${ }^{5}$

$$
\mu_{d p}=\frac{16 \rho e u_{l}^{2} \hbar^{3}}{3 E_{D}^{2} k_{B} T m^{* 2} b} \frac{1}{J_{\mathrm{DP}}(k)} .
$$

In Eq. (15), $\rho$ is the crystal density, $u_{l}$ is the longitudinal acoustic phonon velocity, and $E_{D}$ is the deformation potential. Here, the factor $b$ is called as the related Fang-Howard expression $^{28}$ for the triangular well and given by ${ }^{18}$

$$
b=\left(\frac{33 e^{2} m^{*} n_{2 \mathrm{D}}}{8 \varepsilon_{0} \varepsilon_{s} \hbar^{2}}\right)^{1 / 3},
$$

and $J_{\mathrm{DP}}(k)$ is the integral

$$
J_{\mathrm{DP}}(k)=\int_{0}^{2 k} \frac{1}{2 \pi k^{3}\left(q+q_{s}\right)^{2} \sqrt{1-\left(\frac{q}{2 k}\right)^{2}}} q^{4} d q .
$$

In Eq. (16), $n_{2 \mathrm{D}}$ is the sheet carrier density of the 2DEG which is denoted with $n_{1}$ in Eq. (1). In Eq. (17), the $q_{s}$ is the two dimensional reciprocal screening length which is defined as

$$
q_{s}=\frac{e^{2} m^{*}}{2 \pi \hbar^{2} \varepsilon_{0} \varepsilon_{s}} F_{11}(q) f(0) .
$$

Here, $f(0)$ is the occupation probability at the subband edge. In this study, it can be assumed that all screening is determined by the lowest subband electrons. ${ }^{5}$ Therefore, the occupation probability at the subband edge will be unity. $F_{11}(q)$ is the form factor of ground state Fang-Howard wave function. $^{28}$

The expression for the piezoelectric scattering limited mobility is ${ }^{5}$

$$
\mu_{p e}=\frac{\pi \varepsilon_{0} \varepsilon_{s} \hbar^{3} k}{e K^{2} k_{B} T m^{* 2}} \frac{1}{J_{\mathrm{PE}}(k)},
$$

where, the angular dependence is neglected for simplicity. The integral $J_{\mathrm{PE}}(k)$ is in form

$$
J_{\mathrm{PE}}(k)=\int_{0}^{2 k} \frac{F_{11}(q)}{4 k^{2}\left(q+q_{s}\right)^{2} \sqrt{1-\left(\frac{q}{2 k}\right)^{2}}} q^{3} d q .
$$

c. Background impurity scattering. Scatterings of 2DEG carriers by impurities may investigated in two parts; (i) an ionized impurity scattering due to remote donors and (ii) background impurity scattering due to charges near to interface. Ionized impurity scattering due to remote donors is effective in modulation-doped structures, however background impurity scattering is effective in all structures. In this study, there is no modulation doping as all our samples are nominally undoped. Therefore, we only consider the background impurity scattering. The mobility due to the background impurity scattering is given as ${ }^{29}$

$$
\mu_{\mathrm{BI}}=\frac{8 \pi \hbar^{3} \varepsilon^{2} k_{F}^{2} I_{B}(\beta)}{e^{3} m^{* 2} N_{\mathrm{IMP}}},
$$

where $\varepsilon$ is the dielectric permittivity of $\mathrm{GaN}, k_{F}$ is the wavevector on the Fermi surface, and $N_{\text {IMP }}$ is the impurity density due to background impurities. Here, $N_{\text {IMP }}$ is equal to impurity density that used in Eq. (3). The integral in Eq. $(21), I_{B}(\beta)$ is defined as

$$
I_{B}(\beta)=\int_{0}^{\pi} \frac{\sin ^{2} \theta}{(\sin \theta+\beta)^{2}} d \theta,
$$

where

$$
\beta=S_{0} / 2 k_{F} \text {. }
$$

Here, $S_{0}$ is the screening constant for degenerate case which is given as ${ }^{30}$

$$
S_{0}=\frac{e^{2} m^{*}}{2 \pi \varepsilon \hbar^{2}} \text {. }
$$

d. Interface roughness scattering. Interface roughness can lead to the perturbation of electron energy in a quantum well. Therefore, it is important, specifically, in narrow quantum wells. Narrow pseudotriangular quantum wells of strained $\mathrm{Al}_{\mathrm{x}} \mathrm{Ga}_{1-\mathrm{x}} \mathrm{N} / \mathrm{GaN}$ based heterostructures may have large fluctuations in quantized electron energies because of interface roughness induced by strain relaxation at the related interface. The mobility associated with interface roughness scattering is given as ${ }^{19}$

$$
\mu_{\mathrm{IFR}}=\left(\frac{2 \varepsilon_{0} \varepsilon_{s}}{n_{2 \mathrm{D}} \Delta \Lambda}\right)^{2} \frac{\hbar^{3}}{e^{3} m^{* 2}} \frac{1}{J_{\mathrm{IFR}}(k)} .
$$

Here, $\Delta$ is the lateral size of the roughness and $\Lambda$ is the correlation length between fluctuations. The integral $J_{\mathrm{IFR}}(k)$ in is in form

$$
J_{\mathrm{IFR}}(k)=\int_{0}^{2 k} \frac{e^{-q^{2} \Lambda^{2} / 4}}{2 k^{3}\left(q+q_{s}\right)^{2} \sqrt{1-\left(\frac{q}{2 k}\right)^{2}}} q^{4} d q,
$$

where $q_{s}$ is the screening constant

$$
q_{s}=\frac{e^{2} m^{*}}{2 \pi \hbar^{2} \varepsilon_{0} \varepsilon_{s}} F(q) .
$$

The form factor $F(q)$ in Eq. (26) is given by Hirakawa and Sakaki as ${ }^{31}$

$$
F(q)=\int_{0}^{\infty} d z \int_{0}^{\infty} d z^{\prime}[f(z)]^{2}\left[f\left(z^{\prime}\right)\right]^{2} e^{-q\left|z-z^{\prime}\right|} .
$$

\section{RESULTS AND DISCUSSION}

Figure 1 shows the temperature dependence of Hall mobilities $\left(\mu_{H}\right)$ and Hall sheet carrier densities $\left(n_{H}\right)$ of the investigated samples at $1.4 \mathrm{~T}$ in the temperature range of $T$ $=22$ and $350 \mathrm{~K}$. Within the experimental accuracy the Hall mobility may be accepted as temperature independent under $T=100 \mathrm{~K}$. Sheet carrier density is also nearly temperature independent within the range of measurement temperatures. 


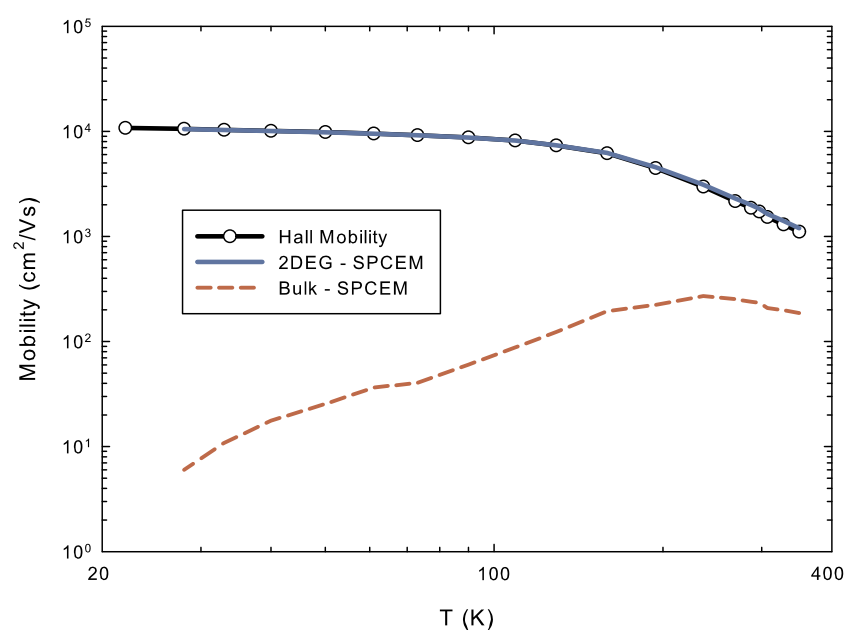

FIG. 1. (Color online) Temperature dependent Hall mobility and Hall sheet carrier densities of the investigated samples.

At high temperatures, sheet carrier density tends to increase due to the increase in the density of thermally induced bulk carriers. $^{32}$ At room temperature and under $1.4 \mathrm{~T}$ magnetic field, Hall mobility and sheet carrier density of the investigated samples are $1724 \mathrm{~cm}^{2} / \mathrm{V} \mathrm{s}$ and $7.95 \times 10^{12} \mathrm{~cm}^{-2}$, respectively. At $22 \mathrm{~K}$, electron mobility is calculated as high as $10766 \mathrm{~cm}^{2} / \mathrm{V} \mathrm{s}$.

To calculate 2DEG and bulk contributions, SPCEM analysis is carried out with using the low magnetic field $(0.05 \mathrm{~T})$ and high magnetic field $(1.4 \mathrm{~T})$ Hall data as the input. SPCEM results are shown in Fig. 2. Mobility of both 2DEG and bulk contributions which are shown in Fig. 2(a) are influenced by the polar optical phonon scattering at high temperatures. ${ }^{27}$ The bulk mobility decreases with decreasing temperature as expected because of the dominating ionized impurity scattering at low temperatures. ${ }^{33}$ In Fig. 2(b) sheet carrier densities of both 2DEG and bulk contributions are presented. 2DEG density is taken as temperature independent as pointed out above and the bulk carriers are gradually frozen out with the decreasing temperature. Fitted calculation for two-donor system is also shown on Fig. 2(b). Here, it is assumed that the bulk carriers are due to donors with binding energies of $\mathrm{E}_{\mathrm{D} 1}=39 \mathrm{meV}$ and $\mathrm{E}_{\mathrm{D} 2}=8 \mathrm{meV}$ as commonly accepted and reported by other groups. ${ }^{1,20,34}$

In order to calculate total impurity density which influences the bulk mobility at lower temperatures, we implement a scattering analysis on temperature dependent bulk mobilities. A successful bulk scattering analysis based on ionized impurity scattering, polar optical phonon scattering, and acoustical phonon scattering mechanisms are shown in Fig. 3. Using Matthiessen's rule, the total mobility is then calculated as the combination of individual mobilities. It is seen that ionized impurity scattering is dominant up to $200 \mathrm{~K}$. Mobility which is limited by acoustic phonon scattering is $>20000 \mathrm{~cm}^{2} / \mathrm{V} \mathrm{s}$ in the whole studied temperature range. Therefore, the effects of acoustic phonon scattering are very small. At high temperatures, the LO-phonon scattering is dominant as expected in a highly polar material as GaN. From the scattering analysis, the momentum relaxation time

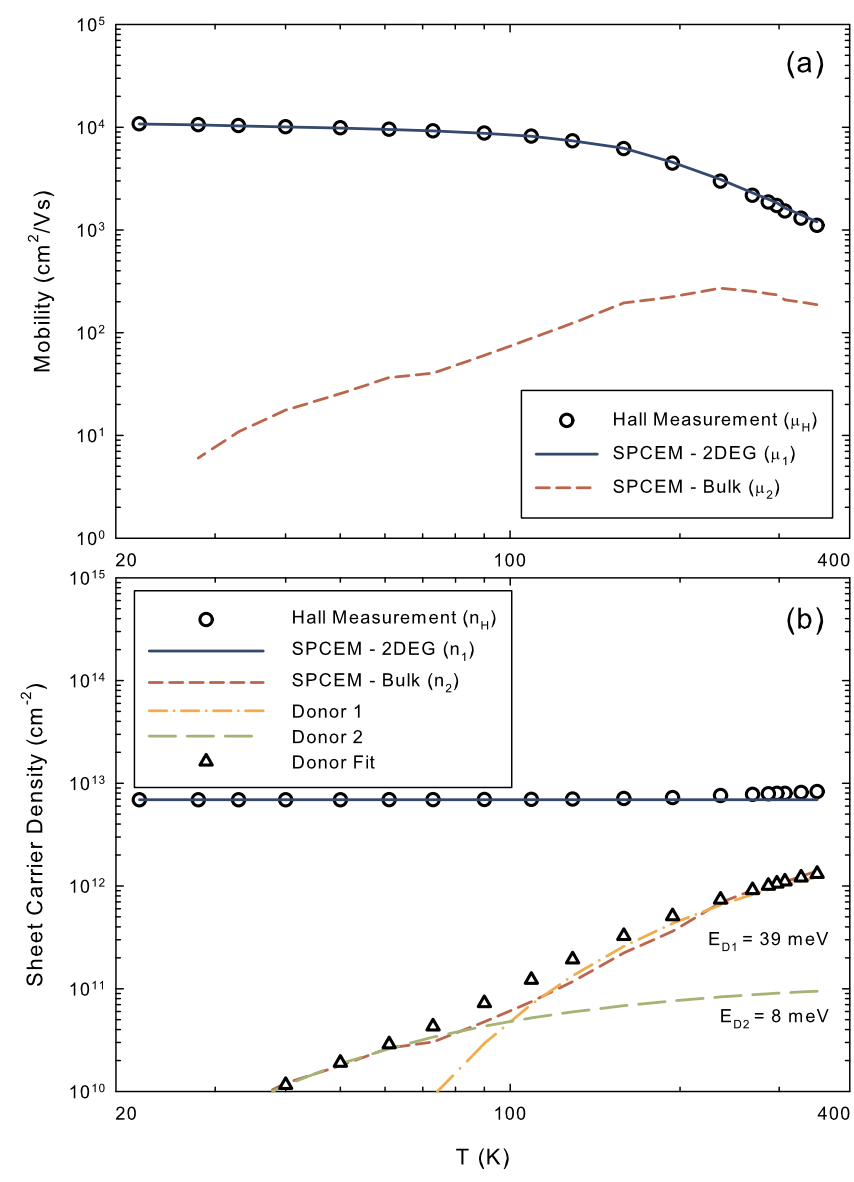

FIG. 2. (Color online) (a) Hall mobilities and (b) sheet carrier densities of the 2DEG and bulk carriers extracted using SPCEM. Fit of donor binding energies for a two-donor system is also shown.

for LO-phonons, deformation potential constant and ionized impurity concentration are determined as $1.05 \times 10^{-13} \mathrm{~s}, 5.0$ $\mathrm{eV}$, and $1.7 \times 10^{23} \mathrm{~m}^{-3}$, respectively.

In order to investigate the $2 \mathrm{D}$ conduction with scattering analysis, mobilities limited by the individual scattering mechanisms, polar optical phonon scattering, acoustic phonon scattering, background impurity scattering, and interface roughness scattering, were calculated from the expressions given in Sec. III B 2. Because all our samples have a $1.5 \mathrm{~nm}$

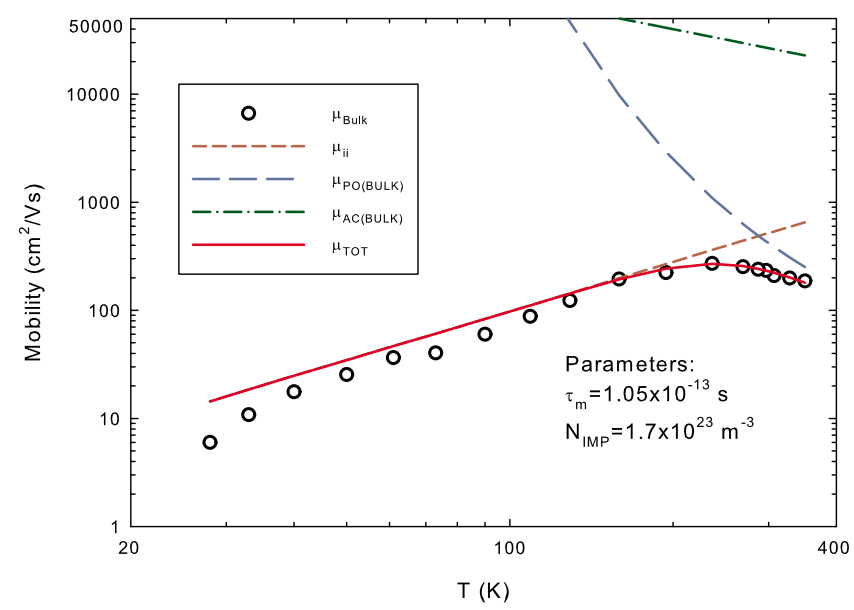

FIG. 3. (Color online) Scattering analysis of temperature dependent mobility of the bulk carrier (carrier 2) extracted using SPCEM. 


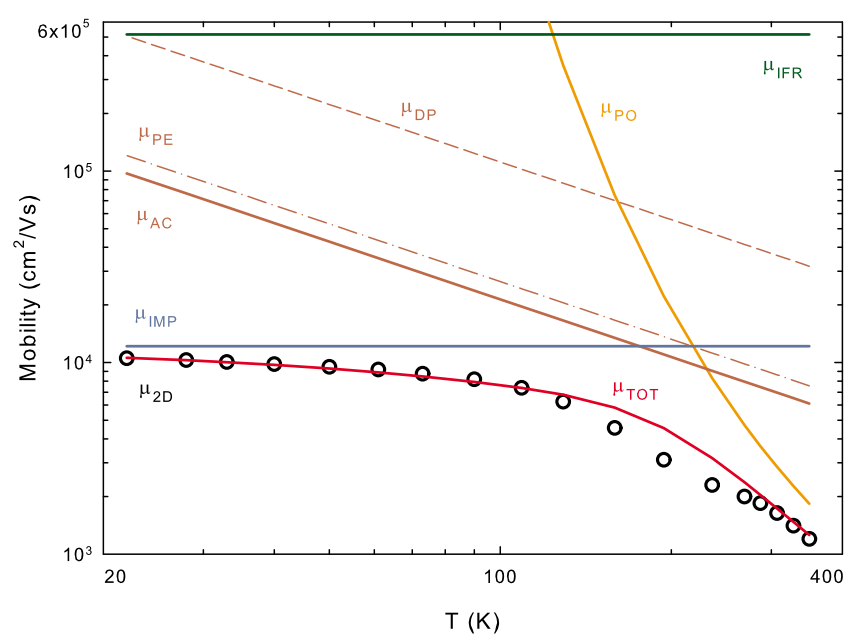

FIG. 4. (Color online) Scattering analysis of temperature dependent mobility of the 2DEG carrier (carrier 1) extracted using SPCEM.

AlN interlayer at the 2DEG interface, the alloy scattering is not taken into account. ${ }^{24}$ In the calculation of background impurity scattering, we used the impurity density value that is obtained as a result of the bulk scattering analysis, for the background impurity scattering. In addition, in 2D conduction scattering analysis, we used the deformation potential value, which is also obtained from the bulk scattering analysis. With these assumptions we have achieved to reduce the fitting parameters for the 2D conduction scattering analysis from four to two. Figure 4 shows the scattering analysis result for the 2D conduction. The agreement between the fitted and measured results is excellent. Here we used as the effective width of pseudotriangular quantum well, $Z_{0}$ $=2.0 \pm 0.05 \mathrm{~nm}$, and correlation length of the interface roughness $\lambda=35.0 \pm 0.24 \mathrm{~nm}$ for the accepted lateral size of $\Delta=2 \mathrm{ML}$. Instead of interface roughness scattering, background impurity scattering appears to be the dominant scattering mechanism at low temperatures in the studied samples. The scattering results of $2 \mathrm{DEG}$ carriers are in agreement with our previous findings. ${ }^{35}$

\section{CONCLUSION}

In this study, Hall effect measurements on unintentionally doped $\mathrm{Al}_{0.22} \mathrm{Ga}_{0.78} \mathrm{~N} / \mathrm{AlN} / \mathrm{GaN} / \mathrm{AlN}$ heterostructures, grown by MOCVD, were carried out as a function of temperature $(22-350 \mathrm{~K})$ and magnetic field $(0.05$ and $1.4 \mathrm{~T})$. Magnetic-field dependent Hall data were analyzed by using the SPCEM technique. With implementing SPCEM, bulk and 2DEG carrier densities and mobilities are extracted successfully. A bulk scattering analysis based on ionized impurity scattering, polar optical phonon scattering, and acoustical phonon scattering mechanisms is performed to SPCEM extracted bulk carrier data. A scattering analysis based on polar optical phonon scattering, acoustic phonon scattering, background impurity scattering, and interface roughness scattering for the 2DEG carrier are performed. In these analyses, we reduced the number of fitting parameters from four to two by combining the bulk and 2D scattering parameters, and we showed that the scattering analyses of the bulk conduction and the 2D conduction consistently in the same sample. Fit parameters and the overall results of both scattering analyses are in excellent agreement with the literature and our previous findings. Implementing this procedure to a known semiconductor thin film will supply important information not only about the carriers, and also information about the phonon interactions, impurities, interface, and quantum well of the related thin film with a simple singlefield temperature dependent electrical measurement. The effects of growth conditions, doping, and layer thicknesses on the parameters of these interactions can be investigated with the help of this easy procedure. Thus, a standardized usage of this procedure may both help the thin film production, and the improvement of this procedure itself.

\section{ACKNOWLEDGMENTS}

This work is supported by the State Planning Organization of Turkey under Grant No. 2001K120590, by the European Union under the projects EU-PHOME and EUECONAM, and TUBITAK under the Project Nos. 106E198, 107A004, and 107A012. One of the authors (Ekmel Ozbay) acknowledges partial support from the Turkish Academy of Sciences.

${ }^{1}$ O. Ambacher, J. Phys. D 31, 2653 (1998).

${ }^{2}$ L. F. Eastman and U. K. Mishra, IEEE Spectrum 39, 28 (2002).

${ }^{3}$ S. Contreras, W. Knap, E. Frayssinet, M. L. Sadowski, M. Goiran, and M. Shur, J. Appl. Phys. 89, 1251 (2001).

${ }^{4}$ L. Hsu and W. Walukiewicz, Phys. Rev. B 56, 1520 (1997).

${ }^{5}$ B. K. Ridley, B. E. Foutz, and L. F. Eastman, Phys. Rev. B 61, 16862 (2000).

${ }^{6}$ S. Gokden, Physica E (Amsterdam) 23, 114 (2004)

${ }^{7}$ J. Antoszewski, M. Gracey, J. M. Dell, L. Faraone, T. A. Fisher, G. Parish, Y.-F. Wu, and U. K. Mishra, J. Appl. Phys. 87, 3900 (2000).

${ }^{8}$ A. Teke, S. Gökden, R. Tülek, J. H. Leach, Q. Fan, J. Xie, Ü. Özgür, H. Morkoç, S. B. Lisesivdin, and E. Özbay, New J. Phys. 11, 063031 (2009).

${ }^{9}$ S. B. Lisesivdin, S. Acar, M. Kasap, S. Ozcelik, S. Gokden, and E. Ozbay, Semicond. Sci. Technol. 22, 543 (2007).

${ }^{10}$ S. B. Lisesivdin, S. Demirezen, M. D. Caliskan, A. Yildiz, M. Kasap, S. Ozcelik, and E. Ozbay, Semicond. Sci. Technol. 23, 095008 (2008).

${ }^{11}$ Z. Dziuba, J. Antoszewski, J. M. Dell, L. Faraone, P. Kozodoy, S. Keller, B. Keller, S. P. DenBaars, and U. K. Mishra, J. Appl. Phys. 82, 2996 (1997).

${ }^{12}$ I. Vurgaftman, J. R. Meyer, C. A. Hoffman, D. Redfern, J. Antoszewski, L. Faraone, and J. R. Lindemuth, J. Appl. Phys. 84, 4966 (1998).

${ }^{13}$ S. B. Lisesivdin, N. Balkan, and E. Ozbay, Microelectron. J. 40, 413 (2009).

${ }^{14}$ M. J. Kane, N. Apsley, D. A. Anderson, L. L. Taylor, and T. Kerr, J. Phys. C 18, 5629 (1985).

${ }^{15}$ S. P. Tobin, G. N. Pultz, E. E. Krueger, M. Kestigian, K. K. Wong, and P. W. Norton, J. Electron. Mater. 22, 907 (1993).

${ }^{16}$ W. A. Beck and J. R. Anderson, J. Appl. Phys. 62, 541 (1987).

${ }^{17}$ J. R. Meyer, C. A. Hoffman, F. J. Bartoli, D. J. Arnold, S. Sivananthan, and J. P. Faurie, Semicond. Sci. Technol. 8, 805 (1993).

${ }^{18}$ J. H. Davies, The Physics of Low-Dimensional Semiconductors (Cambridge University Press, Cambridge, 1998), p. 364.

${ }^{19}$ D. Zanato, S. Gokden, N. Balkan, B. K. Ridley, and W. J. Schaff, Semicond. Sci. Technol. 19, 427 (2004).

${ }^{20}$ D. Zanato, S. Gokden, N. Balkan, B. K. Ridley, and W. J. Schaff, Superlattices Microstruct. 34, 77 (2003).

${ }^{21}$ J. D. Wiley, in Semiconductors and Semimetals, edited by R. K. Wilardson and A. C. Beer (Academic, New York, USA), Vol. 10, p. 32.

${ }^{22}$ N. Balkan, M. C. Arikan, S. Gokden, V. Tilak, B. Schaff, and R. J. Shealy, J. Phys.: Condens. Matter 14, 3457 (2002).

${ }^{23}$ M. Ramonas, A. Matulionis, and L. Rota, Semicond. Sci. Technol. 18, 118 (2003).

${ }^{24}$ S. B. Lisesivdin, A. Yildiz, and M. Kasap, Optoelectron. Adv. Mater. Rapid Commun. 1, 467 (2007).

${ }^{25}$ M. J. Kearney and A. I. Horrell, Semicond. Sci. Technol. 13, 174 (1998). 
${ }^{26}$ H. M. Ng, D. Doppalapudi, T. D. Moustakas, N. G. Weimann, and L. F. Eastman, Appl. Phys. Lett. 73, 821 (1998).

${ }^{27}$ B. K. Ridley, J. Phys. C 15, 5899 (1982).

${ }^{28}$ F. F. Fang and W. E. Howard, Phys. Rev. Lett. 16, 797 (1966).

${ }^{29}$ K. Hess, Appl. Phys. Lett. 35, 484 (1979).

${ }^{30}$ K. Lee, M. S. Shur, T. J. Drummond, and H. Morkoc, J. Appl. Phys. 54, 6432 (1983).

${ }^{31}$ K. Hirakawa and H. Sakaki, Phys. Rev. B 33, 8291 (1986).
${ }^{32}$ S. B. Lisesivdin, A. Yildiz, S. Acar, M. Kasap, S. Ozcelik, and E. Ozbay, Appl. Phys. Lett. 91, 102113 (2007).

${ }^{33}$ M. Shur, B. Gelmont, and M. Asif Khan, J. Electron. Mater. 25, 777 (1996).

${ }^{34}$ W. Götz, N. M. Johnson, C. Chen, H. Liu, C. Kuo, and W. Imler, Appl. Phys. Lett. 68, 3144 (1996).

${ }^{35}$ S. B. Lisesivdin, E. Arslan, M. Kasap, S. Ozcelik, and E. Ozbay, J. Phys.: Condens. Matter 20, 045208 (2008). 